\title{
UMA ANÁLISE SOBRE STARTUPS DE BASE TECNOLÓGICA A PARTIR DA LITERATURA DO PERÍODO 2008-2017
}

Pablo Peron De Paula ${ }^{1}$

Josivânia Silva Farias ${ }^{1}$

Carlos Denner Dos Santos Júnior ${ }^{1}$

${ }^{1}$ Universidade de Brasília 


\section{UMA ANÁLISE SOBRE STARTUPS DE BASE TECNOLÓGICA A PARTIR DA LITERATURA DO PERÍODO 2008-2017}

Resumo: O objetivo desta pesquisa foi analisar as publicações que envolvem startups nos últimos 10 aons e compreender como essas empresas estão sendo estudadas, propondo assim uma agenda de pesquisa. Foram selecionados 84 artigos da base Scientific Periodicals Eletronic Library (SPELL). Os dados coletados foram tratados em três etapas. Primeiramente, descreveu-se o cenário das publicações e a tipificação metodológica dos estudos. Em seguida, através do software Iramuteq realizou-se uma análise exploratória do corpus de texto formado pelos resumos dos artigos. Finalmente, foi realizada uma análise de conteúdo a partir dos objetivos de pesquisa constantes dos artigos. Os resultados mostraram a prevalência de trabalhos qualitativos, realizados através de estudos de caso, tendo a entrevista como instrumento de coleta de dados e a análise de conteúdo como principal técnica de tratamento. A análise reflexiva das categorias e subcategorias proporcionou um modelo teórico além da identificação de uma agenda de pesquisa.

Palavras-chave: Startup. Base tecnológica. Bibliometria. Análise de Conteúdo. 


\section{Introdução}

As Startups podem ser consideradas empresas em fase inicial de suas atividades e que desenvolvem produtos com base tecnológica, portanto a inovação tecnológica está nos princípios de sua estratégia competitiva. Entre os principais aspectos das startups estão, a forma de organização temporária com potencial de rápido crescimento, a atuação em um ambiente de incerteza, e a busca por um modelo de negócios que possa tornar-se repetível e escalável (Blank, 2013). Uma startup é uma instituição designada a entregar um novo produto ou serviço sob condições de extrema incerteza (Ries, 2011). O termo startup tem sido usado de maneira ampla, porém não muito clara, em diversos contextos. Contudo, as definições apresentadas levam a entender que startups são empresas cuja estratégia organizacional e de negócios é sustentada pela inovação e cuja base técnica de produção está sujeita a mudanças frequentes, advindas de ambientes turbulentos e incertos e da concorrente busca por esforços contínuos em pesquisa e desenvolvimento tecnológico.

As startups têm proporcionado novos negócios de base inovadora e tecnológica, e assim, conferido uma alavancagem para a economia dos países (Miranda, Santos Júnior, \& Dias, 2016) e contribuindo significativamente para a criação de empregos em setores de alta tecnologia (Padrão \& Andreassi, 2013). Apesar disso, as startups estão suscetíveis a fracassarem devido à sua vulnerabilidade inicial, considerando que enfrentam mais riscos, possuem menos recursos e têm pouca legitimidade (Miranda et al., 2016). Entretanto, a amarga realidade é que a maioria das startups fracassa, a maioria dos novos produtos não faz sucesso e a maior parte dos empreendimentos não alcança seu potencial máximo (Ries, 2011).

Apesar de originalmente ser um lócus de estudo, as startups podem também ser entendidas como um fenômeno de grande crescimento em diversos países. Esse entendimento se reflete na diversidade em que as pesquisas às têm abordado. Essas pesquisas têm contribuído para compreender as startups sob diferentes prismas como, por exemplo, estudos que abarcam o papel das incubadoras como entidade de fomento (Santos, Dutra, Almeida, \& Sbragia, 2008), os que questionam a efetividade dos modelos de gestão e do processo de incubação (Lobosco, Maccari, Da Costa, \& De Almeida, 2015), os que analisam as práticas de geração de valor (da Rosa, Schreiber, Schmidt, \& Kuhn Junior, 2017), os que buscam compreender a inserção em mercados internacionais (Mais, Castro de Carvalho, Amal, \& Gaia Hoffmann, 2010), os que debatem os diferentes critérios de avaliação de EBTs (Inácio Júnior, Carvalho, \& Gavira, 2013), e os que apontam a relevância e a contribuição da aprendizagem organizacional na inovação de produtos (Rocha \& Vieira, 2016).

Diante desse cenário, surge a seguinte questão: como as startups de base tecnológica estão sendo estudadas e quais as principais lacunas de pesquisa referentes à essas empresas? Assim, essa pesquisa teve como objetivo analisar os estudos sobre startups na busca da compreensão desse fenômeno através de uma bibliometria e de uma análise de conteúdo realizada a partir da base SPELL no período entre 2008 e 2017 e propor uma agenda de pesquisa para estudos futuros.

\section{0 ambiente das Startups de base tecnológica}

A capacidade de um país de desenvolver e difundir inovação em uma área específica é restrita pelas redes de articulações produtivas e tecnológicas entre as empresas, as universidades e os órgãos estatais de pesquisa e fomento à inovação. Fiates, Martins, Fiates,

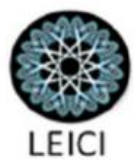


Martignago, e dos Santos (2013), destacam que a inovação é uma vantagem competitiva que impulsiona o processo de internacionalização e é fundamental para o desempenho competitivo da empresa no mercado. A capacidade de inovação das empresas, imersas em um ambiente competitivo global, pode ser considerada um dos fatores-chave na busca por vantagens competitivas e sobrevivência organizacional (de Oliveira, Scherer, de Moura Carpes, Hahn, \& Pivetta, 2016).

No Brasil, entre as ações de fomento à inovação e o desenvolvimento tecnológico está a promoção de EBTs, especialmente quando inseridas em ambiente de incubadoras (Iacono, De Almeida, \& Nagano, 2011). Fiates et al. (2013), ressaltam que EBTs são essencialmente inovadoras e que as incubadoras de empresas se constituem em ambientes potencializadores de redes de relacionamento, tanto entre as empresas incubadas e graduadas, como com agentes externos. Ao fomentar a consolidação de tais redes, as incubadoras passam a estimular a convergência do conhecimento, por meio de fatores endógenos e exógenos ao ambiente de incubação e, dessa forma, as EBTs podem usufruir dessas relações (Tumelero, dos Santos, Marins, \& Carnaúba, 2011).

Em sua pesquisa, Sarquis, Pizzinatto, Sehnem, Pizzinatto, e Duarte (2017) apontam para a existência de facilitadores e dificultadores originários tanto do ambiente externo quanto interno que influenciam os resultados de startups. Dentre esses fatores, no ambiente interno, destacam-se a disponibilidade de mão de obra especializada, a cooperação entre áreas funcionais, o conhecimento de tendências/oportunidades de mercado, as deficiências na estruturação do processo de inovação no método de avaliação/desempenho das inovações e no conhecimento sobre gestão de projetos. No ambiente externo, alguns fatores de influência identificados são existência de fontes de fomento/financiamento externo, parceria com universidade, parceria com instituições do setor/pesquisa, rápida evolução tecnológica no mercado e a deficiência na participação de clientes nos projetos de inovação (Sarquis et al., 2017). Assim, as EBTs imersas em ambientes hipercompetitivos e de rápidas mudanças devem utilizar ferramentas de apoio à gestão da inovação tecnológica (Pitassi, 2012) e estabelecer um modelo de negócio capaz de suportar suas estratégias na busca da obtenção de vantagem competitiva.

\section{Percurso Metodológico}

A abordagem qualitativa foi utilizada neste estudo e, para o alcance dos objetivos propostos, adotou-se como recurso metodológico a bibliometria, que, segundo Araújo e Alvarenga (2011), assume importante papel quando da análise da produção científica em determinada área do conhecimento. Na definição do percurso metodológico foram desenvolvidas 11 etapas, utilizando a proposta de Guerra, Tondolo, e Camargo (2016) de forma adaptada.

$\mathrm{Na}$ etapa 1 foi definido o tema. Assim, emergiu a oportunidade de estudar as Startups de base tecnológica devido à sua crescente importância no desenvolvimento de tecnologias, geração de emprego e renda. Posteriormente, foram realizadas, na etapa 2, as leituras flutuantes. Essa etapa teve como propósito familiarizar o autor com os principais termos relacionados às Startups e ao seu ambiente. Foram lidas as seguintes obras: The Lean Startup How today's entrepreneurs use continuous innovation to create radically successful businesses (Ries, 2011) e, The four steps to the epiphany: successful strategies for products

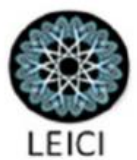


that win (Blank, 2013). Já na etapa 3, foi definido o problema de pesquisa, a saber, como as startups de base tecnológica estão sendo estudadas e quais as principais lacunas de pesquisa referentes a essas empresas?

$\mathrm{Na}$ etapa 4 foi identificada a base de dados, Scientific Periodicals Eletronic Library (SPELL), para a pesquisa no período de 2008 a 2017. A etapa 5 identificou os artigos, para tanto, procedeu-se à busca pela ocorrência das palavras-chave "Startup", "Start-up", "Start up" e "base tecnológica" no título dos artigos. A etapa 6, constituiu-se da seleção dos artigos, assim, na primeira fase, foram selecionados 91 artigos. Contudo, foram retirados 7 artigos que não tinham aderência, restando assim, 84 artigos de 41 diferentes periódicos acadêmicos, publicados em português, espanhol e inglês e revisados por pares.

A etapa 7 constituiu-se da análise descritiva-exploratória do material coletado. Essa etapa dividiu-se em 2 fases. Primeiramente, todos os artigos selecionados foram submetidos à análise das seguintes dimensões: nome do periódico, ano de publicação e; enquadramento teórico. Os artigos identificados como teóricos empíricos, foram ainda analisados quanto aos seguintes critérios: abordagem metodológica; natureza do estudo; instrumento de coleta de dados e; técnicas de análise. Esses dados foram analisados a partir do uso da estatística descritiva e apresentados através de gráficos e tabelas destacando o cenário descritivo da produção acadêmica que envolve as startups.

Posteriormente, foi constituído um corpus de análise a partir dos resumos das 84 publicações. Esse corpus foi explorado através de uma análise da nuvem de palavras, e da classificação hierárquica descendente (CHD) com auxílio do software IRAMUTEQ. O Interface de $R$ pour les Analyses Multidimensionnelles de Textes et de Questionnaires (IRAMUTEQ) é um software gratuito, ancorado no ambiente estatístico do software $\mathrm{R}$ e desenvolvido na linguagem python. O programa identifica e reformata unidades de textos, transformando Unidades de Contexto Iniciais (UCI) em Unidades de Contexto Elementares (UCE) para utilizar em suas análises lexicais (Camargo \& Justo, 2013).

A nuvem de palavras permite uma visualização lexicográfica das palavras mais representativas do corpus investigado e pode ser utilizada na fase de pré-análise, servindo de base para se elaborar o corpus que estará sujeito à análise de conteúdo (Lemos, 2016). O método da CHD classifica os segmentos de textos em função de seus respectivos vocabulários, e o conjunto deles é dividido com base na frequência das formas lematizadas. Essa análise permite obter classes de UCE que, ao mesmo tempo, apresentam vocabulário semelhante entre si e diferente das UCE das outras classes (Camargo \& Justo, 2013). A CHD foi utilizada para que emergisse do corpus analisado as categorias a posteriori.

$\mathrm{Na}$ etapa 8 foi realizada uma análise de conteúdo, seguindo as etapas de préanálise, exploração do material e tratamento dos resultados, conforme proposto por Bardin (1977). Dos 84 artigos, foram selecionados os resumos para constituírem o corpus a ser analisado. Em seguida, deu-se início a codificação que, segundo Bardin (1977) corresponde a uma transformação, a partir de regras bem definidas, do corpus selecionado em uma representação de conteúdo. Na categorização, foi utilizado o critério semântico, no qual os objetivos com os mesmos significados foram agrupados de forma conjunta. As categorias foram definidas a posteriori, a partir da CHD e da nuvem de palavras realizadas no IRAMUTEQ. A elaboração das categorias buscou atender os critérios de exclusão mútua, homogeneidade, pertinência, objetividade e fidelidade e produtividade (Bardin, 1977; Franco,

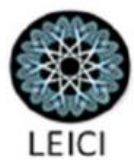


2005).

Na etapa 9 foi construído o referencial teórico, a partir da síntese da análise da nuvem de palavras, da análise fatorial de correspondência e da classificação hierárquica descendente (etapa 7) e da codificação dos objetivos (etapa 8). Na etapa 10 foi desenvolvida a análise e discussão dos resultados (seção 4) e, finalizando, na etapa 11, foram apresentadas as considerações finais, agenda de pesquisa e limitações do trabalho (seção 5).

\section{Resultados}

Pontuados os aspectos conceituais e metodológicos da pesquisa, esta seção faz referência aos resultados do levantamento dos 84 artigos analisados, bem como apresenta uma discussão quanto à análise lexical e semântica do conteúdo e proposição de uma agenda de pesquisa para a área.

\subsection{Mapeando os estudos}

No que tange à evolução dos estudos que envolvem as Startups de base tecnológica, identificou-se significativo aumento na produção científica no período, demonstrado pela curva de tendência linear, conforme demonstrado na Figura 1. Esse crescimento fica evidenciado quando se compara o número de publicações dos 5 primeiros anos do período analisado, 2008 a 2012, com os últimos 5 anos, 2013 a 2017. Os primeiros 5 anos totalizaram 34 publicações que representa $40,5 \%$ do total, enquanto os últimos 5 anos somaram 50 publicações, representando 59,5\%. O ano de 2016 se destaca com 18 publicações, representando $21,4 \%$ das publicações.

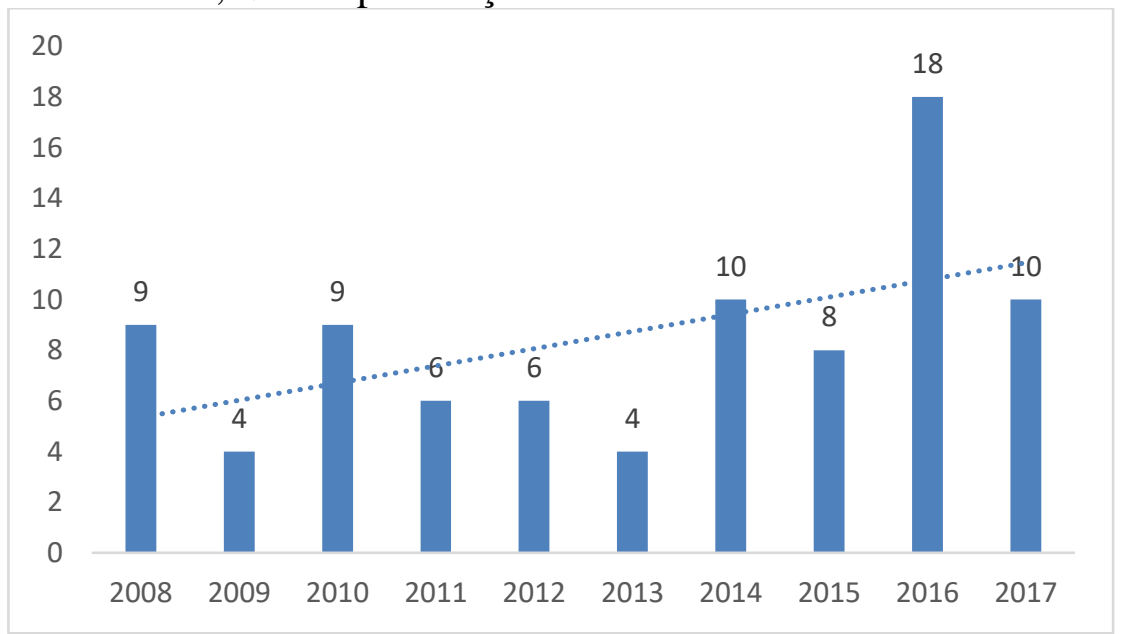

Figura 1: Evolução das publicações no período de 2008 a 2017.

Analisando a produção científica em relação aos periódicos de publicação, constatou-se que, no período de análise, 41 diferentes periódicos publicaram pesquisas que envolviam Startups de base tecnológica. A Revista de Administração e Inovação (RAI) foi responsável por 19,0\% dos estudos publicados. Também obteve destaques a Revista Gestão \& Tecnologia, a Revista de Negócios e a Revista de Empreendedorismo e Gestão de Pequenas Empresas. Percebe-se que os periódicos que mais publicam pesquisas envolvendo Startups de base tecnológica têm em seus escopos temas como inovação, tecnologia, negócios e 
empreendedorismo, que são centrais no lócus estudado. Essa observação pode ser reforçada na missão da RAI, a saber, "divulgar a produção intelectual na área de inovação tecnológica, organizacional e de mercado, estimulando as contribuições criativas e inéditas do trabalho acadêmico e de pesquisa" (Revista de Administração e Inovação, 2017).

\subsection{Tipificação metodológica dos estudos analisados}

Em se tratando dos aspectos metodológicos e do enquadramento dos estudos, a Tabela 1 ilustra os tipos e a natureza das pesquisas realizadas. Os resultados demonstram a predominância de trabalhos teórico-empíricos $(95,2 \%)$, na busca de atestar conceitos e reforçar teorias. Quanto à natureza, verifica-se que 76,3\% dos artigos classificam-se como pesquisa qualitativa, $18,8 \%$ como quantitativas e 5,0\% como multimétodo. Os trabalhos classificados como multimétodo incluem, entre outros, os estudos de Gallon e Ensslin (2008), na busca por analisar o potencial de liderança criativa e; Iacono et al. (2011), que procuram avaliar o grau de importância das interações e relações de cooperação das startups no período de incubação, assim como a tendência desse comportamento para o período de pós-incubação.

Tabela 1 - Tipificação metodológica dos estudos

\begin{tabular}{llc}
\hline Análise Metodológica & Classificação & Frequência \\
\hline Tipo de pesquisa & Teórico-Empírico & $95,2 \%$ \\
& Ensaio Teórico & $4,8 \%$ \\
\hline Natureza da Pesquisa & Qualitativo & $76,3 \%$ \\
& Quantitativo & $18,8 \%$ \\
& Multimétodo & $5,0 \%$ \\
\hline Estratégia de abordagem & Estudo de caso & $60,0 \%$ \\
& Exploratório-descritiva & $25,0 \%$ \\
& Survey & $16,3 \%$ \\
& Pesquisa ação & $3,8 \%$ \\
& Fenomenologia & $1,3 \%$ \\
& Grounded Theory & $1,3 \%$ \\
\hline Instrumento de coleta de dados & Entrevista & $58,8 \%$ \\
& Questionário & $36,6 \%$ \\
& Documentos & $27,5 \%$ \\
& Levantamento bibliográfico & $13,8 \%$ \\
& Dados secundários & $7,5 \%$ \\
& Observação & $5,0 \%$ \\
& Grupo Focal & $1,3 \%$ \\
\hline Técnicas de análise & Análise de Conteúdo & $71,3 \%$ \\
& Estatística Descritiva & $26,3 \%$ \\
& Análise Documental & $3,8 \%$ \\
& Análise Fatorial & $3,8 \%$ \\
& Modelagem de Equações Estruturais & $3,8 \%$ \\
& Estatística Inferencial & $2,5 \%$ \\
& Outros & $11,3 \%$ \\
\hline
\end{tabular}

Ainda na Tabela 1, verifica-se a estratégia de abordagem metodológica, os instrumentos de coleta de dados e as técnicas de análise. Nesse sentido, identifica-se que a maioria absoluta das pesquisas realizadas $(60,0 \%)$, utilizou-se de estudo de casos únicos ou múltiplos como estratégia de abordagem. Na sequência aparecem os estudos exploratório- 
descritivos, com 25,0\%, seguidos de surveys, que representam 16,3\% dos estudos. A pesquisa-ação foi utilizada em $3,8 \%$ das pesquisas e, completam as abordagens, a fenomenologia e a grounded theory, ambas com $1,3 \%$ dos estudos.

Quanto aos instrumentos de coleta de dados, destacam-se a utilização das entrevistas $(58,8 \%)$, dos questionários $(36,6 \%)$, documentos $(27,5 \%)$ e o levantamento bibliográfico $(7,5 \%)$ dos estudos analisados. As bases de dados secundárias $(7,5 \%)$, a observação $(5,0 \%)$ e o grupo focal $(1,3 \%)$ foram menos utilizados. Nesse quesito, vale destacar que a utilização de mais de uma fonte de dados pode ocorrer no caso de pesquisas com mais de uma etapa (Machado, Selig, Follmann, \& Casarotto Filho, 2016) ou mesmo como forma de triangulação multimétodo (Sperafico, Engelman, \& Gonçalves, 2017).

Por fim, dentre as técnicas de análise de dados, o principal destaque é a análise de conteúdo, utilizada em $71,3 \%$ dos estudos, que cada vez mais vem sendo empregada para fazer inferências acerca de dados textuais (Franco, 2005), seguida da estatística descritiva (26,3\%). A análise de documentos, a análise fatorial e a modelagem de equações estruturais foram utilizadas em apenas 3,8\% dos estudos analisados e, finalmente, a estatística inferencial aparece como técnicas de análise em somente 2,5\% das pesquisas. Outras técnicas de pesquisas como correlação, regressão linear, regressão logística e análise de redes sociais foram aplicadas apenas uma vez cada, somando um total de 11,3\%.

\subsection{Explorando os estudos de Startups}

A análise da representatividade da abordagem em comum presente nos estudos foi possível por meio do método da nuvem de palavras. A formação lexicográfica das palavras com maior representatividade, conforme a Figura 2, são, "empresa", "como", "inovação", "incubadora", "pesquisa" e, "conhecimento". A partir dessas palavras entende-se que as startups estão sendo estudadas sobre diferentes aspectos e, como o principal intuito de entender e descrever esse fenômeno. Essa observação é reforçada pela expressão adverbial de modo "como", que teve um destaque significativo e expressa o caráter exploratório das pesquisas.

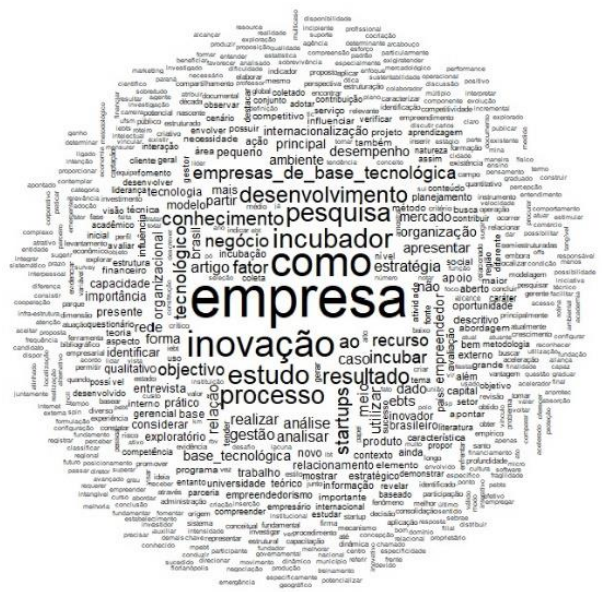

Figura 2: Nuvem de palavras dos resumos dos 84 artigos estudados.

Com o objetivo de verificar o agrupamento dos segmentos de textos do corpus analisado, foi realizada a classificação hierárquica descendente (CHD), considerando cada artigo como uma unidade de contexto inicial (UCI). Desse modo, o corpus geral, composto

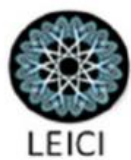


por 84 textos, resultou em 448 segmentos de texto (ST), com aproveitamento de 364 STs (81,25\%). Emergiram 15.670 ocorrências (palavras, formas ou vocábulos), sendo 2.860 palavras distintas e 1.524 com ocorrência única. O conteúdo analisado foi categorizado em quatro classes: Classe 1, com 78 ST (21,43\%); Classe 2, com 81 ST (22,25\%); Classe 3 com 103 ST $(28,30 \%)$; e Classe 4 com 102 ST $(28,02 \%)$. Foram consideras as variáveis ativas e ilustrativas com $\chi^{2} \geq 3,97(\mathrm{p}<0,05)$ para a análise.

As quatro classes se encontram divididas em três ramificações do corpus total em análise. A primeira ramificação é formada pela Classe 4, aqui denominada como "Método", e se distingue das três demais classes que derivam de outra ramificação. As classes derivadas de uma mesma ramificação apresentam diferenças, mas também guardam semelhanças entre si. Assim, as Classes 1 e 2 guardam alguma semelhança entre si e, pelas formas que as compõem podem ser identificadas como "Objetivos" e "Apoio" respectivamente. A Classe 3, apesar de ser distinta das Classes 1 e 2, guarda alguma relação com estas e podem ser "Fatores" necessários ao sucesso das startups. As classes e as principais palavras que as compõem estão representadas na Figura 3.

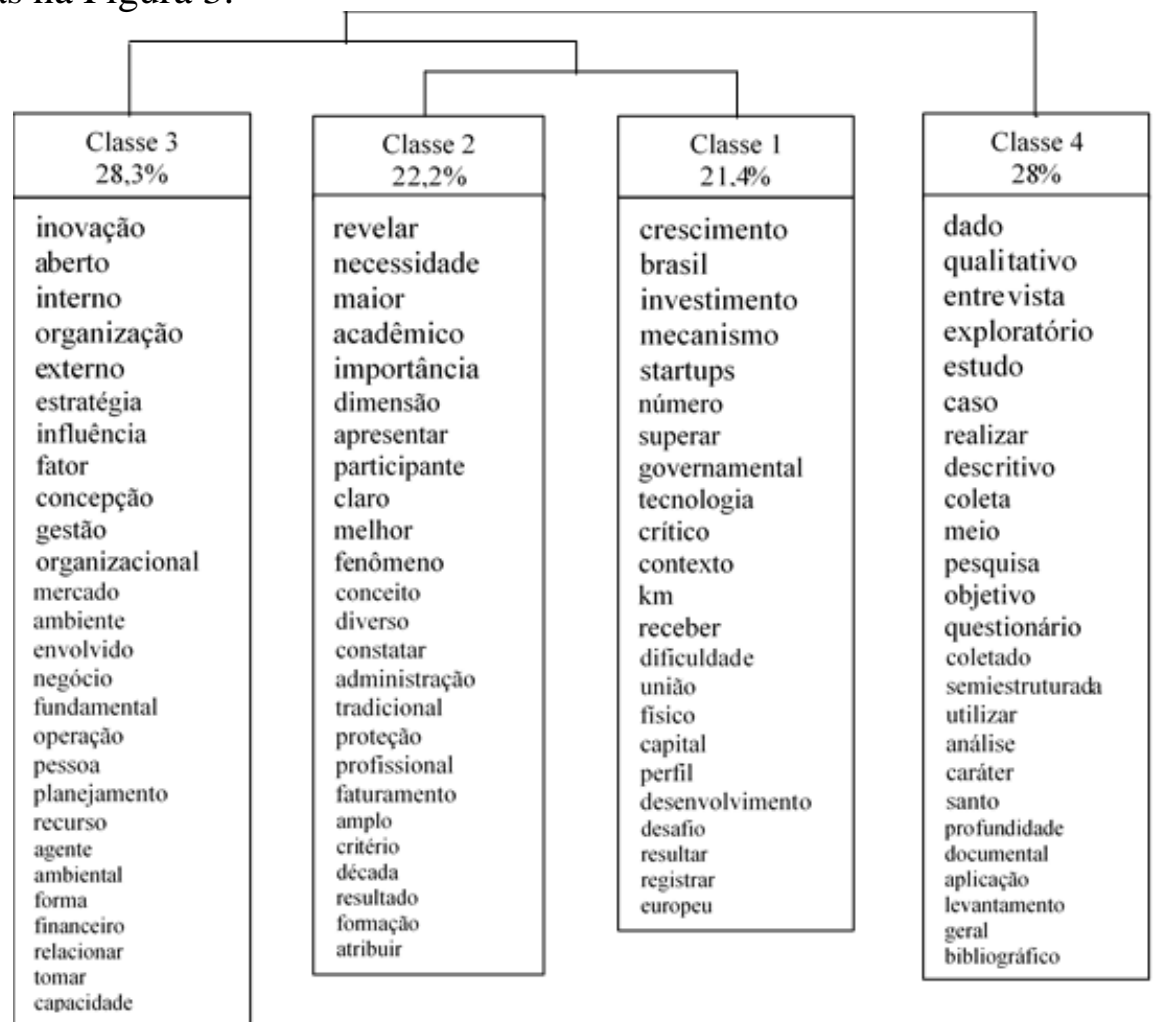

Figura 3 - Dendograma para os resumos dos 84 artigos investigados.

\subsection{Sintetizando os estudos sobre Startups}

A Tabela 2 apresenta as categorias, subcategorias, síntese dos objetivos e as referências que deram o suporte teórico à análise de conteúdo. 
Tabela 2 - Análise de conteúdo dos objetivos

\begin{tabular}{|c|c|c|c|}
\hline Categorias & Subcategorias & Síntese dos objetivos & Referências \\
\hline \multirow[t]{3}{*}{ Ambiente } & Estratégia & $\begin{array}{l}\text { Formular e analisar estratégias, os seus processos de formação e a } \\
\text { sua relação com o desempenho de EBTs. }\end{array}$ & $\begin{array}{l}\text { Berté, Rodrigues, e Almeida (2008); da Silva, Escrivão Filho, e } \\
\text { Terence (2009); Sanches e Machado (2013). }\end{array}$ \\
\hline & $\begin{array}{c}\text { Iniciativa } \\
\text { Empreendedora }\end{array}$ & $\begin{array}{c}\text { Identificar, descrever e analisar a intenção, os sinais e as práticas } \\
\text { empreendedoras bem como a influência destas na captação de } \\
\text { recursos e no desempenho. }\end{array}$ & $\begin{array}{l}\text { Fonsêca, Mello, Marçal, e Correa (2008); Piscopo (2010); Paiva } \\
\text { Jr e Fernandes (2012); Martins, Fiates, Dutra, da Silva Leite, e } \\
\text { Giarola (2014); Santiago, Barbosa, Cheng, Reis, e Jamil (2014); } \\
\text { Campos, Parellada, Quintero, e Valenzuela (2015); Tumelero, } \\
\text { dos Santos, e Kuniyoshi (2016). }\end{array}$ \\
\hline & $\begin{array}{c}\text { Redes de } \\
\text { Relacionamentos }\end{array}$ & $\begin{array}{c}\text { Descrever e analisar as redes de relacionamento, avaliar as interações } \\
\text { e a influência no desempenho. }\end{array}$ & $\begin{array}{l}\text { Gallon, Ensslin, e Silveira (2009); Mais et al. (2010); Paiva Jr, } \\
\text { Fernandes, e Almeida (2010); (Iacono et al., 2011). }\end{array}$ \\
\hline \multirow[t]{5}{*}{ Recursos } & Conhecimento & $\begin{array}{l}\text { Identificar as práticas e mecanismos de compartilhamento e } \\
\text { convergência de conhecimento para superar fatores críticos. }\end{array}$ & $\begin{array}{l}\text { Campos, Parellada, de la Parra, e Palma (2011); Dalmarco, } \\
\text { Maehler, Trevisan, e Schiavini (2017). }\end{array}$ \\
\hline & Estrutura & $\begin{array}{c}\text { Analisar, avaliar e classificar a estrutura organizacional, os fatores } \\
\text { que contribuem e a sua relação com as competências. }\end{array}$ & $\begin{array}{l}\text { Ferreira, Marques, e Fernandes (2010); Lima, de Macêdo, } \\
\text { Cabral, e Colares (2014); Silva e Reis (2015). }\end{array}$ \\
\hline & $\begin{array}{l}\text { Incubadora / } \\
\text { Aceleradora }\end{array}$ & $\begin{array}{l}\text { Analisar as formas de apoio, o nível de desenvolvimento dos } \\
\text { processos de incubação e sua influência no desempenho das EBTs. }\end{array}$ & $\begin{array}{l}\text { Serra, Serra, Ferreira, e Fiates (2011); Barbosa e Hoffmann } \\
\text { (2013). }\end{array}$ \\
\hline & Gestão & $\begin{array}{l}\text { Descrever e difundir o processo e as práticas de planejamento, e a } \\
\text { gestão dos recursos necessários à concretização dos objetivos. }\end{array}$ & $\begin{array}{l}\text { Maehler, Cassanego Júnior, e Schuch Júnior (2009); Reis, } \\
\text { Cheng, Ladeira, e Fernandes (2014); Alves Silva (2016). }\end{array}$ \\
\hline & $\begin{array}{l}\text { Gestão de Pessoas } \\
\text { \& Liderança }\end{array}$ & $\begin{array}{c}\text { Identificar práticas de gestão de pessoas, programas de treinamento e } \\
\text { desenvolvimento e analisar o perfil de liderança em EBTs. }\end{array}$ & $\begin{array}{l}\text { Gallon e Ensslin (2008); Matos e Quelhas (2007); Sala e } \\
\text { Trevisan (2014). }\end{array}$ \\
\hline \multirow[t]{4}{*}{ Resultados } & Desempenho & $\begin{array}{l}\text { Investigar indicadores de avaliação, analisar os fatores que } \\
\text { influenciam e comparar o desempenho entre EBTs. }\end{array}$ & Miranda et al. (2016). \\
\hline & Inovação & $\begin{array}{l}\text { Compreender as características, propor procedimentos que } \\
\text { proporcionem, analisar os mecanismos utilizados e avaliar programas } \\
\text { e processos de inovação. }\end{array}$ & $\begin{array}{l}\text { Rodrigues, Heringer, e França (2010); Pitassi (2012); Desidério } \\
\text { e Oliveira (2012); De Paula, Starling, Nascimento, e Barbosa } \\
\text { (2015). }\end{array}$ \\
\hline & Internacionalização & $\begin{array}{l}\text { Analisar as características do processo de internacionalização e os } \\
\text { fatores que contribuem para o sucesso desse processo. }\end{array}$ & $\begin{array}{l}\text { D'Elia e Zouain (2008); Ribeiro, Oliveira Jr, e Borini (2012); } \\
\text { Viana (2012). }\end{array}$ \\
\hline & $\begin{array}{c}\text { Modelos Teóricos e } \\
\text { de Negócios }\end{array}$ & $\begin{array}{l}\text { Analisar e propor modelos de negócios e formatar um modelo teórico } \\
\text { de enquadramento para EBTs. }\end{array}$ & Inácio Júnior et al. (2013); Mello, Fonseca, e Vieira (2012). \\
\hline
\end{tabular}


A primeira categoria, Ambiente, é composta pelas subcategorias Estratégia, Iniciativa Empreendedora e Redes de Relacionamento. Essas três subcategorias representam um conjunto de fatores que podem ser considerados elementos necessários para o surgimento de startups.

A segunda categoria, Recursos, é composta pelas subcategorias Conhecimento, Estrutura, Incubadora / Aceleradora, Gestão e Gestão de Pessoas \& Liderança. Essas cinco subcategorias representam a base de recursos necessária para a sobrevivência das startups e para a proliferação desse tipo de empresa em uma determinada região.

A terceira categoria, Resultados, é composta pelas subcategorias Desempenho, Inovação, Internacionalização e Modelos Teóricos e de Negócios. Essas quatro subcategorias podem ser entendidas como os objetivos a serem alcançados pelas startups.

\subsection{Uma breve discussão dos resultados.}

A análise dos artigos sobre startups, realizada no período de 2008 a 2017, propicia o desenvolvimento de uma agenda de pesquisa que permita uma melhor compreensão do universo no qual estão inseridas essas empresas. Os resultados desta pesquisa geram proposições de agenda que envolve uma maior interação entre o lócus e as teorias organizacionais e maior diversificação entre os métodos adotados.

Primeiramente, no que tange aos métodos, percebe-se uma concentração de trabalhos de natureza qualitativa, que utilizaram a estratégia do estudo de caso, com coleta de dados através de entrevista e que empregaram como técnica a análise de conteúdo. Esse cenário se explica pelo fato de as startups serem um fenômeno recente e com grande crescimento em diversos países. Dessa forma, os pesquisadores podem buscar compreender o fenômeno investigando de forma profunda as suas características e, assim, realizar proposições e levantar hipóteses. Contudo, a partir daqui, torna-se necessária a diversificação dos métodos, com a inclusão de pesquisas de natureza quantitativa, na qual surveys seriam uma importante estratégia e através da coleta de dados com questionários aplicados a amostras estatisticamente significativas, pudessem ser analisados por técnicas como a regressão múltipla, a regressão logística e a modelagem de equações estruturais para a confirmação de hipóteses e de modelos teóricos.

O caráter exploratório das pesquisas que abordam as startups no período de 2008 a 2017 pode ser reforçado pelo destaque da expressão adverbial de modo "como" na nuvem de palavras. A constante utilização desse advérbio demonstra que as startups podem ser consideradas lócus ou fenômeno ainda pouco compreendido. A nuvem de palavras também destaca os termos: "empresa", "inovação", "base tecnológica", "conhecimento", "fator", "estratégia", dentre outros. A compreensão desses termos deu origem a algumas subcategorias de análise. A Classificação Hierárquica Descendente deu origem a quatro classes. Uma dessas classes está relacionada aos métodos de pesquisa utilizados e já discutidos anteriormente. As três classes restantes estão relacionadas com as startups em si, e a tudo que pode estar presente no contexto dessas empresas. Essa análise estatística agrupou as palavras mais frequentes nos resumos dos artigos de acordo com as semelhanças lexicais dos segmentos de textos gerados. Assim, a partir do corpo de palavras das classes e a análise interpretativa do pesquisador, as classes que emergiram foram nomeadas como: "Apoio", "Fatores" e
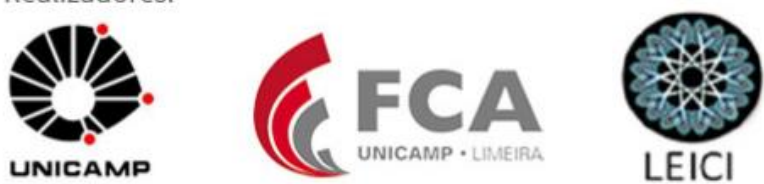
"Objetivos".

Finalmente, os termos que emergiram da nuvem de palavras e a reflexão sobre as classes sugeridas na CHD, serviram de suporte para a construção de categorias e subcategorias na análise de conteúdo. As categorias foram definidas como: "Ambiente", "Recursos" e, "Resultados". Na categoria "Ambiente", foram estabelecidas as seguintes subcategorias: "Estratégia", "Iniciativa Empreendedora" e, "Redes de Relacionamentos". Na categoria "Recursos", encontram-se as seguintes subcategorias: "Conhecimento", "Estrutura", "Incubadora / Aceleradora", "Gestão", "Gestão de Pessoas \& Liderança". Por fim, na categoria "Resultados", ocorre a presença das subcategorias: "Desempenho", "Inovação", "Internacionalização" e, "Modelos Teóricos e de Negócios". Uma visão sistêmica da análise de conteúdo possibilitou propor que para o surgimento de startups, tornam-se necessários alguns fatores ambientais. A ocorrência desses fatores com a disponibilidade e boa utilização de recursos proporcionará às startups maior probabilidade de atingir seus resultados, conforme o modelo representado na Figura 4.

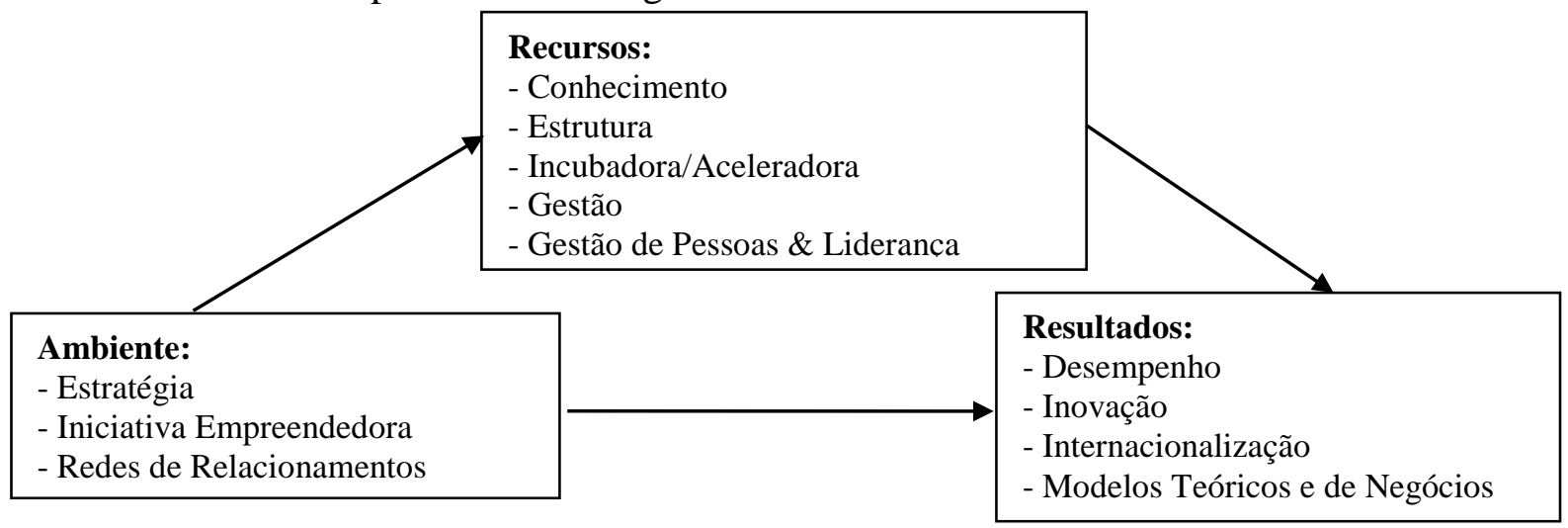

Figura 4 - Modelo teórico-hipotético dos fatores que impactam os resultados de startups.

Considerando o papel primordial da gestão dos recursos para o alcance dos objetivos e o fato de as startups estarem inseridas em ambientes incertos, essa pesquisa estabelece como agenda um aprofundamento dos estudos de startups sob a ótica da Teoria da Visão Baseada em Recursos. Considerando que as startups são empresas inseridas em um contexto de inovação e incerteza, um passo seguinte seria o aprofundamento dos estudos através da perspectiva da Teoria das Capacidades Dinâmica, definida por Teece, Pisano, e Shuen (1997) como a "capacidade para interagir, construir e reconfigurar competências internas e externas para lidar com ambientes que mudam rapidamente".

\section{Considerações Finais}

Essa pesquisa buscou compreender o fenômeno das startups através de uma análise bibliométrica, retratando o cenário atual dos estudos produzidos no período entre 2008 e 2017. Os resultados dos trabalhos permitiram identificar que há um significativo crescimento na produção científica que abordam as startups, bem como traçar o perfil dos estudos, descrever a tipificação metodológica e construir categorias de análise. 
Certifica-se que os estudos sobre startups têm seu ápice no ano de 2016 e a Revista de Administração e Inovação é a grande incentivadora nacional das publicações dessas pesquisas. Apesar da predominância dos estudos qualitativos, dos estudos de casos e das análises de conteúdo, começam a surgir, nos últimos cinco anos, trabalhos quantitativos envolvendo a utilização de técnicas como a Modelagem de Equações Estruturais e a Análise Fatorial Exploratória. Esse fato, embora ainda pouco frequente, demostra ser um indício de que os pesquisadores começaram a buscar comprovações estatísticas de teorias e modelos hipotéticos.

A proposição de uma agenda de pesquisa para os estudos que envolvem startups atenta para a diversificação dos métodos utilizados, tanto na estratégia de abordagem quanto na coleta e tratamento dos dados. Essa agenda busca também, aprofundar os estudos sob a ótica de duas teorias que podem ser consideradas complementares, a Teoria da Visão Baseada em Recursos e a Teoria das Capacidades Dinâmicas. Dessa forma, a proposição da agenda sugere investigar a relação entre ambiente, recursos e resultados nas startups de base tecnológicas.

Em que pesem o interesse e a iniciativa na realização do trabalho, não se pode deixar de destacar a ocorrência de limitações. Elementos como relação com outras temáticas e teorias poderiam enriquecer ainda mais os resultados. A utilização de apenas uma base de busca (SPELL) também é considerada um fator limitante. Assim, sugere-se expandir as buscas para bases como Portal Periódicos da CAPES, Ebsco Host, Emerald Insight, Oxford Journals, Sage Journals, Scielo Brasil, Science Direct (Elsevier), Spell, Springer Link, Taylor \& Francis, Wiley Online Library tornando a pesquisa mais sistematizada. Para tanto, torna-se necessário aprimorar os termos de busca, uma vez que o termo "Startup" possui múltiplos sentidos na língua inglesa.

Além das exposições colocadas, este estudo não se caracteriza como conclusivo, mas como uma prerrogativa para oportunidade de novos trabalhos, um esforço inicial de investigação para a consolidação e direcionamento dos estudos sobre startups no campo da administração. Considerando a relevância do tema, faz-se necessária a contínua atualização e aprofundamento, de forma a preencher as tantas lacunas, conceituais, metodológicas, métricas e subjetivas que perfazem a temática.

\section{Referências}

Alves Silva, S. (2016). Por que analisar a gestão das incubadoras de empresas de base tecnológica sob a ótica da Resource-Based View? REAd-Revista Eletrônica de Administração, 22(3).

Araújo, R. F., \& Alvarenga, L. (2011). A bibliometria na pesquisa científica da pós-graduação brasileira de 1987 a 2007. Encontros Bibli: revista eletrônica de biblioteconomia e ciência da informação, 16(31).

Barbosa, L. G. d. F., \& Hoffmann, V. E. (2013). Incubadora de empresas de base tecnológica: percepção dos empresários quanto aos apoios recebidos. RAI Revista de Administração e Inovação, 10(3), 206-229.

Bardin, L. (1977). Análise de Conteúdo. Tradução de Luiz Antero Reto e Augusto Pinheiro. Lisboa: Ed, 70.

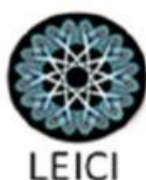


Berté, É. C. O. P., Rodrigues, L. C., \& Almeida, M. I. (2008). A formulação de estratégias para pequenas empresas de base tecnológica. Revista de Administração da Universidade Federal de Santa Maria, 1(1).

Blank, S. (2013). The four steps to the epiphany: successful strategies for products that win: BookBaby.

Camargo, B. V., \& Justo, A. M. (2013). IRAMUTEQ: um software gratuito para análisede dados textuais. Temas em Psicologia, 21(2), 513-518.

Campos, H. M., Parellada, F. S., de la Parra, J. P. N., \& Palma, Y. (2011). Empresas de base tecnológica derivadas de la universidad: el vínculo empresario-oportunidad tecnológica. RAI Revista de Administração e Inovação, 8(4), 117-141.

Campos, H. M., Parellada, F. S., Quintero, M. R., \& Valenzuela, F. A. A. (2015). Creative thinking style and the discovery of entrepreneurial opportunities in startups. Revista de Negócios, 20(1), 3-12.

D'Elia, M. A. G., \& Zouain, D. M. (2008). Superação das barreiras técnicas ao comércio internacional pelas pequenas e médias empresas de base tecnológica-a exportação de produtos eletromédicos para a união Européia. RAI-Revista de Administração $e$ Inovação, 5(1), 05-19.

da Rosa, S. C., Schreiber, D., Schmidt, S., \& Kuhn Junior, N. (2017). Management Pratices that Combine Value Cocreation and User Experience: An Analysis of the Nubank Startup in the Brazilian Market. Revista de Gestão, Finanças e Contabilidade, 7(2), 22.

da Silva, S. J. T., Escrivão Filho, E., \& Terence, A. C. F. (2009). Strategic and Operational Planning in Small Business: a study about its influence in technology-based enterprises of São Carlos. Revista de Negócios, 14(1), 30-45.

Dalmarco, G., Maehler, A. E., Trevisan, M., \& Schiavini, J. M. (2017). The use of knowledge management practices by Brazilian startup companies. RAI Revista de Administração e Inovação.

de Oliveira, M. C. S. F., Scherer, F. L., de Moura Carpes, A., Hahn, I. S., \& Pivetta, N. P. (2016). A Influência da Capacidade de Inovação sobre o Desempenho Internacional: Um Estudo com empresas de base tecnológica. Economia \& Gestão, 16(44), 192-212.

De Paula, H. C., Starling, D. B., Nascimento, J. F., \& Barbosa, F. V. (2015). Mensuração da inovação em empresas de base tecnológica. RAI Revista de Administração e Inovação, 12(4), 232-253.

Desidério, P. H. M., \& Oliveira, J. B. (2012). Empreendedorismo e open innovation: uma análise de projetos de base tecnológica.

Ferreira, J. J. d. M., Marques, C. S., \& Fernandes, C. (2010). Teorias de localização aplicadas às empresas de base tecnológica: um estudo empirico. RAI-Revista de Administração e Inovação, 7(4).

Fiates, G. G. S., Martins, C., Fiates, J. E. A., Martignago, G., \& dos Santos, N. (2013). Análise do papel da incubadora na internacionalização de empresas de base tecnológica, incubadas e graduadas. Revista Eletrônica de Estratégia \& Negócios, 6(1), 252-274. 
Fonsêca, F. R. B., Mello, S. C. B. d., Marçal, M. C. C., \& Correa, M. I. d. S. (2008). Jovens Empreendedores estão preparados para empreender em empresas de Base Tecnológica. Revista de Negócios, 13(3), 67-80.

Franco, M. L. P. B. (2005). Análise de conteúdo.

Gallon, A. V., \& Ensslin, S. R. (2008). Potencial de liderança criativa em equipes de trabalho de empresas de base tecnológica incubadas. RAI-Revista de Administração $e$ Inovação, 5(1).

Gallon, A. V., Ensslin, S. R., \& Silveira, A. (2009). Rede de relacionamentos em pequenas empresas de base tecnológica (EBTs) incubadas: um estudo da sua importância para o desempenho organizacional na percepção dos empreendedores. JISTEM: Journal of Information Systems and Technology Management, 6(3).

Guerra, R. M. d. A., Tondolo, V. A. G., \& Camargo, M. E. (2016). O Que (Ainda) Podemos Aprender sobre Capacidades Dinâmicas. Revista Ibero-Americana de Estratégia, 15(1), 44.

Iacono, A., De Almeida, C. A. S., \& Nagano, M. S. (2011). Interação e cooperação de empresas incubadas de base tecnológica: uma análise diante do novo paradigma de inovação. Revista de Administração Pública-RAP, 45(5).

Inácio Júnior, E. I., Carvalho, R. d. Q., \& Gavira, M. (2013). Proposição de um Novo Método de Seleção de Micro, Pequenas e Médias Empresas de Base Tecnológica (MPEBT). REGEPE-Revista de Empreendedorismo e Gestão de Pequenas Empresas, 1(2), 3-32.

Lemos, L. M. P. (2016). Nuvem de tags como ferramenta de análise de conteúdo: uma experiência com as cenas estendidas da telenovela Passione na internet. Lumina, $10(1)$.

Lima, S. M., de Macêdo, J. L., Cabral, A. C. d. A., \& Colares, R. F. (2014). Estrutura organizacional das empresas vinculadas à incubadora de Base Tecnológica da Universidade de Fortaleza: uma análise sob a perspectiva de Mintzberg. REGERevista de Gestão, 21(3), 305-324.

Lobosco, A., Maccari, E. A., Da Costa, P. R., \& De Almeida, M. R. (2015). Aplicabilidade de modelo de negócios em incubadoras de empresas de base tecnológica para sua autossustentabilidade: Um estudo em incubadoras portuguesas. Revista Alcance, 22(4).

Machado, E., Selig, P. M., Follmann, N., \& Casarotto Filho, N. (2016). Structural capital influence analysis in the success of Incubated startups: A research with 21 entrepreneurs. International Journal of Innovation, 4(1), 46.

Maehler, A. E., Cassanego Júnior, P. V., \& Schuch Júnior, V. F. (2009). A universidade e o desenvolvimento de empresas de base tecnológica. Base, 6(1).

Mais, I., Castro de Carvalho, L., Amal, M., \& Gaia Hoffmann, M. (2010). Importância das redes nos processos de inovação e internacionalização de empresas de base tecnológica. RAI-Revista de Administração e Inovação, 7(1).

Martins, C., Fiates, G. G. S., Dutra, A., da Silva Leite, A. L., \& Giarola, P. G. (2014). Empreendedorismo inovador gerado pelas incubadoras de base tecnológica: Mapeamento da produção científica até 2013. Revista de Negócios, 19(2), 86-108.

Matos, S. T., \& Quelhas, O. L. G. (2007). Organizações humanamente sustentáveis: estudo de caso em organizações de base tecnológica. Organização \& Estratégia, 3(3), 277-301.

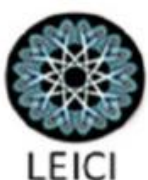


Mello, S. C. B. d., Fonseca, F. R. B., \& Vieira, R. S. G. (2012). Sensemaking e o Desenvolvimento de Redes de Relacionamentos de Negócios no Contexto das Empresas de Base Tecnológica. Organizações \& Sociedade, 19(61).

Miranda, J. Q., Santos Júnior, C. D., \& Dias, A. T. (2016). A influência das variáveis ambientais e organizacionais no desempenho de startups. REGEPE-Revista de Empreendedorismo e Gestão de Pequenas Empresas, 5(1), 28-65.

Padrão, L. C., \& Andreassi, T. (2013). The performance of startups based technology: a comparative study in Brazilian geographic gegions. Revista da Micro e Pequena Empresa, Campo Limpo Paulista, 7(2), 66-79.

Paiva Jr, F. G. d., \& Fernandes, N. C. M. (2012). A contribuição da competência relacional do empreendedor para aperfeiçoar a qualidade de relacionamento entre empresas de base tecnológica. RAI Revista de Administração e Inovação, 9(3), 53-76.

Paiva Jr, F. G. d., Fernandes, N. C. M., \& Almeida, L. (2010). A competência de relacionamento contribuindo para o desenvolvimento de capital social da empresa de base tecnológica. Revista de Negócios, 15(1), 11-28.

Piscopo, M. R. (2010). Empreendedorismo corporativo e competitividade em empresas de base tecnológica. RAI-Revista de Administração e Inovação, 7(1).

Pitassi, C. (2012). Inovação aberta na perspectiva das empresas brasileiras de base tecnológica: proposta de articulação conceitual. RAI Revista de Administração $e$ Inovação, 9(3), 77-102.

Reis, L. P., Cheng, L. C., Ladeira, M. B., \& Fernandes, J. M. (2014). Contribuições ao processo de planejamento de negócio para geração de empresas de base tecnológica de origem acadêmica (EBTS de OA). RAI Revista de Administração e Inovação, 11(4), 732.

Revista de Administração e Inovação, R. (2017). Políticas editoriais: Foco e escopo. Retrieved from https://www.revistas.usp.br/rai/about/editorialPolicies\#focusAndScope

Ribeiro, F. F., Oliveira Jr, M., \& Borini, F. M. (2012). Internacionalização acelerada de empresas de base tecnológica: o caso das Born Globals Brasileiras. RAC-Revista de Administração Contemporânea, 16(6).

Ries, E. (2011). The lean startup: How today's entrepreneurs use continuous innovation to create radically successful businesses: Crown Books.

Rocha, A. d. F. R., \& Vieira, A. M. (2016). Aprendizagem organizacional e inovação de produtos: estudo em empresas de base tecnológica do Vale da Eletrônica (MG). Caderno Profissional de Administração da UNIMEP, 6(1), 108-131.

Rodrigues, L. C., Heringer, B. H. d. F., \& França, A. L. (2010). Padrões de inovação em multinacional de base tecnológica. RAI-Revista de Administração e Inovação, 7(3).

Sala, O. T. M., \& Trevisan, L. N. (2014). A construção de carreira em ambientes inovativosum estudo nas empresas de base tecnológicas. RAI Revista de Administração $e$ Inovação, 11(2), 154-178.

Sanches, P. L. B., \& Machado, A. G. C. (2013). Estratégias de inovação e RBV: evidências em uma empresa de base tecnológica. RAI Revista de Administração e Inovação, 10(4), 183-207.

Santiago, R. A., Barbosa, F. V., Cheng, L. C., Reis, L. P., \& Jamil, G. L. (2014). Um Framework de fatores e esforços determinantes para a captação de recursos financeiros

\section{Organizadores:}
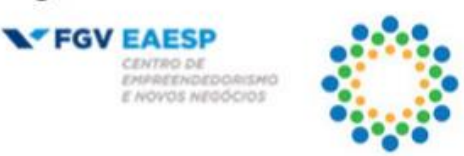

ANEGEPE

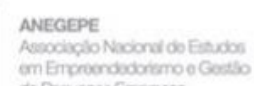

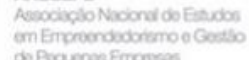

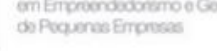

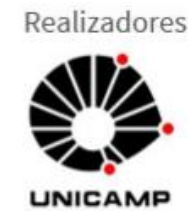

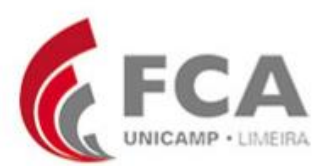


por empresas nascentes de base tecnológica. Revista Base (Administração $e$ Contabilidade) da UNISINOS, 11(1).

Santos, G. D., Dutra, I. S., Almeida, F. R., \& Sbragia, R. (2008). Um estudo sobre indicadores de avaliação de incubadoras de base tecnológica no Brasil. Revista Capital CientíficoEletrônica (RCCee)-ISSN 2177-4153, 6(1), 257-283.

Sarquis, A. B., Pizzinatto, N. K., Sehnem, S., Pizzinatto, A. K., \& Duarte, A. C. (2017). Processo de Inovação, Fatores de Influência e Métricas de Desempenho: Proposta de Modelo Conceitual para Empresa de Base Tecnológica. Revista de Administração da Unimep, 15(1), 73-99.

Serra, B., Serra, F. R., Ferreira, M. P., \& Fiates, G. G. (2011). Fatores fundamentais para o desempenho de incubadoras de base tecnológica. RAI Revista de Administração e Inovação, 8(1), 221-248.

Silva, S. E., \& Reis, L. P. (2015). O processo de estruturação de recursos no contexto de uma empresa de base tecnológica de origem acadêmica (EBTA). RAI Revista de Administração e Inovação, 12(2), 153-179.

Sperafico, J. H., Engelman, R., \& Gonçalves, M. A. (2017). Capital intelectual organizacional e inovação em micro e pequeneas empresas de base tecnológica. RAUnP-ISSN 19844204, 9(1), 51-61.

Teece, D. J., Pisano, G., \& Shuen, A. (1997). Dynamic capabilities and strategic management. In Knowledge and strategy (pp. 77-115): Elsevier.

Tumelero, C., dos Santos, S. A., \& Kuniyoshi, M. S. (2016). Sobrevivência de empresas de base tecnológica pós-incubadas: estudo sobre a ação empreendedora na mobilização e uso de recursos. REGE-Revista de Gestão, 23(1), 31-40.

Tumelero, C., dos Santos, S. A., Marins, C., \& Carnaúba, A. C. (2011). Estudo do conhecimento em empresas de base tecnológica incubadas: proposição de um modelo conceitual integrativo. Revista Gestão \& Tecnologia, 11(1), 2-17.

Viana, R. B. C. (2012). A Estratégia na Incerteza: explorando cenários para empresas recémcriadas no mercado brasileiro de tecnologia da informação. REUNA, 17(3), p. 85-98. 